Research Paper

\title{
Let-7a Is an Antihypertrophic Regulator in the Heart via Targeting Calmodulin
}

Xin Zhou ${ }^{1,2 *}$, Fei Sun ${ }^{1} 3^{*}$, Shenjian Luo ${ }^{1}$, Wei Zhao ${ }^{1}$, Ti Yang ${ }^{1}$, Guiye Zhang1, Ming Gao1, Renzhong Lu1,

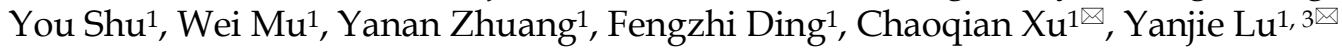

1. Department of Pharmacology (State-Province Key Laboratories of Biomedicine-Pharmaceutics of China, Key Laboratory of Cardiovascular Research, Ministry of Education), College of Pharmacy, Harbin Medical University, Harbin, Heilongjiang 150081, P. R. China.

2. Department of Cardiology (Key Laboratory of Myocardial Ischemia, Ministry of Education), The 2nd Affiliated Hospital of Harbin Medical University, Harbin, Heilongiiang 150081, P. R. China

3. Northern Translational Medicine Research and Cooperation Center, Heilongjiang Academy of Medical Sciences, Harbin Medical University, Harbin, Heilongjiang 150081, P. R. China.

* These authors contributed equally to this work.

$\square$ Corresponding authors: Yanjie Lu (E-mail: yjlu@hrbmu.edu.cn) or Chaoqian Xu (E-mail: xuchaoqian@ems.hrbmu.edu.cn), Department of Pharmacology (the State-Province Key Laboratories of Biomedicine-Pharmaceutics of China), Harbin Medical University, Harbin, Heilongjiang 150081, P.R. China (Fax. +86 451 8667-1354, Tel. +86 451 8667-1354).

(c) Ivyspring International Publisher. This is an open access article distributed under the terms of the Creative Commons Attribution (CC BY-NC) license (https:// creativecommons.org/licenses/by-nc/4.0/). See http://ivyspring.com/terms for full terms and conditions.

Received: 2016.05.27; Accepted: 2016.10.10; Published: 2017.01.01

\begin{abstract}
Background: MicroRNAs (miRNAs) have been emerged as important regulator in a multiple of cardiovascular disease, including arrhythmia, cardiac hypertrophy and fibrosis, and myocardial infarction. The aim of this study was to investigate whether miRNA let-7a has antihypertrophic effects in angiotensin II (AngII)-induced cardiac hypertrophy.

Methods: Neonatal rat ventricular myocytes (NRVMs) were exposed to Angll for $36 \mathrm{~h}$ as a cellular model of hypertrophy; subcutaneous injection of Angll for 2 weeks was used to establish a mouse model of cardiac hypertrophy in vivo study. Cell surface area (CSA) was measured by immunofluorescence cytochemistry; expression of hypertrophy-related genes ANP, BNP, $\beta-M H C$ was detected by Real-time PCR; luciferase activity assay was performed to confirm the miRNA's binding site in the calmodulin ( $\mathrm{CaM}$ ) gene; $\mathrm{CaM}$ protein was detected by Western blot; the hypertrophy parameters were measured by echocardiographic assessment.

Results: The expression of let-7a was decreased in Angll-induced cardiac hypertrophy in vitro and in vivo. Overexpression of let-7a attenuated Angll-induced increase of cell surface area and repressed the increased mRNA levels of ANP, BNP and $\beta-M H C$. Dual-luciferase reporter assay showed that let-7a could bind to the 3'UTR of CaM 1 gene. Let-7a downregulated the expression of $\mathrm{CaM}$ protein. In vivo, let-7a produced inhibitory effects on cardiac hypertrophy, including the downregulation of cross-sectional area of cardiomyocytes in mouse heart, the reduction of IVSD and LVPWD, the suppression of hypertrophy marker genes ANP, BNP, $\beta-M H C$ mRNA level, and the downregulation of CaM protein level.
\end{abstract}

Conclusions: let-7a possesses a prominent anti-hypertrophic property by targeting CaM genes. The findings provide new insight into molecular mechanism of cardiac hypertrophy.

Key words: Cardiac hypertrophy; miRNA; let-7a; angiotensin II; calmodulin.

\section{Introduction}

Cardiac hypertrophy is a physiological or adaptive response that is often associated with increased cell size, protein synthesis and fibroblast proliferation [1-3]. Pathological cardiac hypertrophy can be caused by a number of pathological stimuli, such as hypertension, myocardial infarction, and hypertrophic agents including AngII, isoproterenol and endothelin1 [4]. However, prolonged cardiac 
hypertrophy may lead to heart failure and sudden death $[5,6]$. It has been known that multiple signaling pathways or molecules participate in cardiac hypertrophy [7-9]. A role for CaM has been reported in several physiological and pathological processes in the cardiac hypertrophy [10, 11].

MicroRNAs (miRNAs) are small non-coding RNAs that modulate gene expression by inhibiting translation or promoting degradation of mRNA [12]. Recent studies demonstrated that miRNAs play a critical role in regulation of cardiac developmental and cardiovascular diseases [13-15]. It has been reported that let-7 plays a crucial role in multiple biologic functions, including tumor suppression [16, 17], axon regeneration [18], arrhythmia inhibition [19], and cardiac fibrosis and apoptosis reduction [20]. A study by Gray et al showed that upregulation of let-7 may be involved in regulation of cardiac hypertrophy process in rats [21]. Furthermore, Yang et al reported that upregulation of let-7 induced by thioredoxin 1 attenuates cardiac hypertrophy by targeting cyclin D2 [22].

One of biological functions of miRNA is that single miRNA may have multiple mRNA targets by binding to their 3'untranslated region. By analyzing miRNA target, we found that let-7a and the 3'UTR of the CaM 1 gene have complementary binding sites, indicating CaM gene may be another target of let-7 in regulation of cardiac hypertrophy process. We therefore hypothesized that let-7a might play a role in regulating cardiac hypertrophy by targeting CaM. Thereby, the aim of this study was to examine this hypothesis.

\section{Methods}

\section{Cardiomyocytes culture and treatment}

Briefly, the isolated hearts from 1 to 3-day-old Sprague-Dawley rats were cut into $1.0 \mathrm{~mm}$ pieces and digested in $0.25 \%$ trypsin at $37^{\circ} \mathrm{C}$. Subsequently, cells in the supernatant were isolated by centrifugation (1500 rpm, $5 \mathrm{~min}$ ) at room temperature. The cells were then cultured in DMEM (Hyclone Laboratories, UT, USA) supplemented with $10 \%$ fetal bovine serum (Gibco, Invitrogen, CA, USA) for $2 \mathrm{~h}$. After fibroblast adherence, the cells in the supernatant were seeded onto cell culture dishes. After $48 \mathrm{~h}$ culture, NRVMs were treated with AngII (Sigma-Aldrich Co, St. Louis, $\mathrm{MO}$, USA) $100 \mathrm{nM}$ for $36 \mathrm{~h}$ to induce cell hypertrophy, which has been proven to induce cardiomyocyte hypertrophy [23].

\section{Animal experiments}

Healthy male C57BL/ 6 mice $(20 \pm 2 \mathrm{~g})$ used in the current study were housed under standard animal room conditions (temperature at $21 \pm 1^{\circ} \mathrm{C}$, humidity of
$55 \pm 5 \%)$. Cardiac hypertrophy model was established by AngII administration. The mice were subcutaneously injected with AngII for 2 weeks at a concentration of $1.5 \mathrm{mg} / \mathrm{kg} /$ day. Saline-injected mice served as controls. All animal experiments were approved by the ethics committee of Harbin Medical University.

\section{Synthesis of let-7a mimics and let-7a mimics transfection}

Let-7a mimics (5'UGAGGUAGUAGGUUGUAU AGUU-3'), its anti-miRNA oligonucleotides (AMO-let-7a, 5' -AACUAUACAACCUACUACCUCA $\left.-3^{\prime}\right)$ and scrambled RNA (5'-UUCUCCGA ACGUGUCACGU-3') as a negative control (NC) were synthesized by Ribo Biotechnology Co, Ltd (Guangzhou, China). miRNA transfection was performed using X-tremeGene siRNA Transfection Reagent (Roche, USA). In briefly, $100 \mathrm{nM}$ of let-7a mimics/NC or $200 \mathrm{nM}$ AMO-let-7a were added to the medium at room temperature to form transfection complexes. The cells were incubated in serum-free DMEM with the transfection complexes for $6 \mathrm{~h}$, and then the medium was replaced with DMEM containing $10 \%$ FBS.

\section{Construction and infection of lentivirus}

Lentivirus vectors expressing let-7a, AMO-let-7a, or NC sequence were constructed by Invitrogen Co. (Shanghai, China). Virus-containing solution $(20 \mu \mathrm{L}, 1$ $\times 10^{8} \mathrm{TU}$ ) including len-negative control (len-NC), precursor let-7a (len-pre-let-7a), AMO-let-7a (len-AMO-let-7a) were injected into left ventricular chamber of mouse heart by blocking aorta of short duration.

\section{Measurement of cell surface area}

Cells with various treatments were washed with PBS and fixed with $4 \%$ paraformaldehyde for $15 \mathrm{~min}$ at room temperature. After that, the cells were washed and treated with $0.4 \%$ Triton X-100. After 90 $\mathrm{min}$, the cells were incubated with goat serum at room temperature for $60 \mathrm{~min}$. Then the samples were incubated with anti-sarcomeric actin antibody (Sigma-Aldrich, St. Louis, MO, USA) at $4^{\circ} \mathrm{C}$ overnight. Next day, cells were stained with FITC-conjugate goat anti-mouse antibody for $1 \mathrm{~h}$. Images were obtained under a fluorescence microscope (Nikon 80i, Japan). Cell surface area was determined using Image-Pro Plus Data Analysis Software.

\section{Quantitative Real-time PCR}

Total RNA was extracted from cells or heart tissues using TRIZOL reagent (Invitrogen, USA). The expression levels of ANP, BNP and $\beta$-MHC mRNAs were performed on ABI 7500 fast Real Time PCR 
system (Applied Biosystems, USA) by quantitative real-time PCR. The sequences of ANP, BNP, $\beta$-MHC primers were listed in Table 1 and synthesized by Guangzhou Ribo Biotechnology Co. Ltd (Guangzhou, China). GAPDH served as an internal control. Reaction conditions were set as: $95^{\circ} \mathrm{C}$ for $10 \mathrm{~min}, 40$ cycles of $95^{\circ} \mathrm{C}$ for $15 \mathrm{sec}, 60^{\circ} \mathrm{C}$ for $30 \mathrm{sec}$, and $72^{\circ} \mathrm{C}$ for $30 \mathrm{sec}$. The relative quantitative expression was determined using method $2^{-\wedge} \mathrm{Ct}$.

\section{Dual-luciferase reporter assay}

To confirm that CaM was targeted by let-7a, we constructed a chimeric luciferase reporter system (pMIR-REPORT, Ambion, CA, USA) tagged with the 3'UTR region of rat CaM1 containing the seed match sequence with or without two nucleotide mutations. HEK293 cells were transfected with $200 \mathrm{ng}$ of pMIR-REPORT luciferase vector and 8 ng of Prl-SV40 renilla luciferase control vector in the presence of the let-7a mimics or scrambled-oligonucleotide (as negative control) using Lipofectamine 2000 (Invitrogen, USA). Luciferase activity was assayed after $48 \mathrm{~h}$ using the Dual-luciferase reporter assay system (Promega, USA) normalized to Renilla luciferase activity.

\section{Western blot analysis}

Total protein was extracted from the cardiomyocytes and left ventricular tissues of heart with RIPA lysis buffer (Beyotime Institute of Biotechnology, China). Then, the homogenates were centrifuged at $13,500 \mathrm{rpm}$ for $15 \mathrm{~min}$ at $4^{\circ} \mathrm{C}$. The supernatant was collected and protein concentrations were determined using BCA Protein Assay Kit (Beyotime Institute of Biotechnology, China). The protein samples $(100 \mu \mathrm{g})$ were separated to SDS-PAGE on $10 \%$ polyacrylamide gels, and subsequently transferred to nitrocellulose membrane (Millipore, Bedford, MA, USA), and then blocked

Table 1. The sequence of PCR primers.

\begin{tabular}{ll}
\hline \multicolumn{1}{c}{ Primer } & \multicolumn{1}{c}{ Sequence } \\
\hline GAPDH-F & 5'-TCTACATGTTCCAGTATGACTC-3' \\
GAPDH-R & ''-ACTCCACGACATACTCAGCACC-3' \\
ANP-F & ''-CTCCGATAGATCTGCCCTCTTGAA-3' \\
ANP-R & 5 '-GGTACCGGAAGCTGTTCGAGCCTA-3' \\
BNP-F & 5 '-TTGGGCAGAAGATAGACCGGAT-3' \\
BNP-R & 5 '-GGTCTTCCTAAAACAACCTCA-3' \\
$\beta$-MHC-F & 5 'AACCTGTCCAAGTTCCGCAAGGTG-3' \\
$\beta$-MHC-R & 5 '-GAGCTGGGTAGCACAAGAGCTACT-3' \\
\hline
\end{tabular}

with a solution of skim milk powder for $2 \mathrm{~h}$ at room temperature. The membranes were incubated overnight with the primary antibody for CaM (Abcam, MA, USA) at $4^{\circ} \mathrm{C}$. The next day, the membranes were washed 3 times with PBS-T and conjugated with a secondary rabbit or mouse polyclonal antibody at room temperature for $1 \mathrm{~h}$. Western blot bands were quantified using Odyssey v1.2 software and normalized to GAPDH.

\section{Cardiac hypertrophy measurements}

After 2 weeks of AngII administration, all mice were sacrificed. The mouse hearts were immediately dissected out and weighed. The heart weight (HW), body weight (BW), and left ventricular weight (LVW) were recorded. The $\mathrm{HW} / \mathrm{BW}$ and $\mathrm{LVW} / \mathrm{BW}$ were calculated to estimate the extent of cardiac hypertrophy.

\section{Histological examination}

The mouse hearts were washed with saline and fixed with $10 \%$ formalin. Then the hearts were cut into $7 \mu \mathrm{m}$ slices, and stained with hematoxylin and eosin (H\&E). Cross-sectional images of cardiomyocytes were scanned at $400 \times$ magnification to estimate the degree of cardiomyocyte hypertrophy. Cross-section area of cells was measured with Image Pro-Plus 4.0 system.

\section{Echocardiographic assessment of cardiac function}

The male C57BL/6 mice were lightly anesthetized and cardiac function was evaluated by using Vevo2100 High-Resolution Imaging system (Visualsonics, Toronto, Canada) equipped with a 30 $\mathrm{MHz}$ microscan transducer. M-mode traces were obtained in both parasternal long and short axis views. Ventricular parameters including interventricular septum depth (IVSD), diastolic left ventricular posterior wall thicknesses (LVPWD) and left ventricular internal diastolic diameter (LVIDD) were measured. Fractional shortening (FS) and ejection fraction (EF) were calculated based on the Vevo2100 High-Resolution Imaging system.

\section{Statistical analysis}

All experimental data are expressed as mean \pm SD. Statistical significance was estimated by ANOVA or student's $t$-test for multiple group or two group comparisons, using SPSS 13.0 software. Values of $P<0.05$ were considered to indicate statistically significant differences.

\section{Results}




\section{Downregulation of let-7a in angiotensin II-induced cardiac hypertrophy}

We first established in vitro and in vivo mouse models of cardiac hypertrophy. Neonatal rat ventricular myocytes (NRVMs) were treated with angiotensin II (AngII) $100 \mathrm{nM}$ for $36 \mathrm{~h}$ to induce myocardial hypertrophy characterized by increased cell surface area (CSA) $(P<0.01$, Fig. 1A and 1B). AngII $1.5 \mathrm{mg} / \mathrm{kg} /$ day was subcutaneously injected in mice for 2 weeks to establish cardiac hypertrophy characterized by increased cross-sectional area of cardiomyocytes $(P<0.01$, Fig. 1C and 1D).

In order to investigate whether let-7 is involved

A
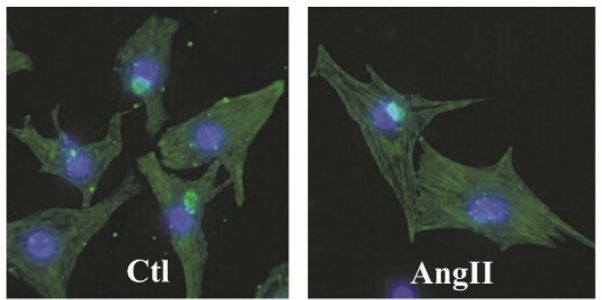

B

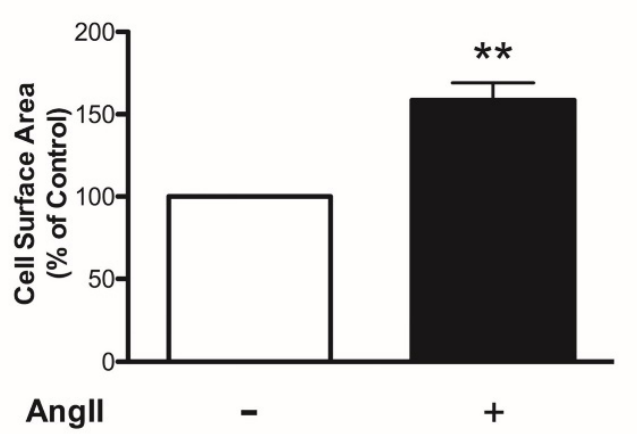

in AngII-induced cardiac hypertrophy, we performed Real-time PCR to quantify the expression of let-7 family miRNAs in cardiac hypertrophic models. The expression of let-7a, let-7d, and let-7e was significantly downregulated in AngII-induced cellular model (Fig. 2A) and mouse model of hypertrophy (Fig. 2B), expression of let-7b and let-7c had no obviously changed. Among them, let-7a showed the most downregulation in expression after AngII treatment both in vivo and in vitro. We therefore chose let-7a as a candidate miRNA in the following experiments.

C
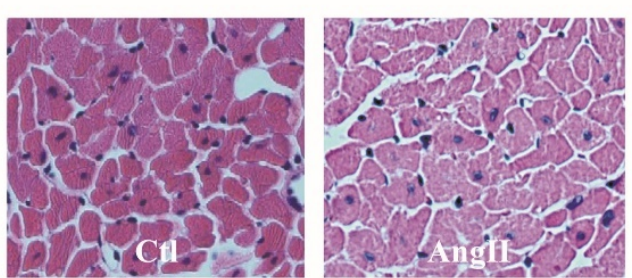

D

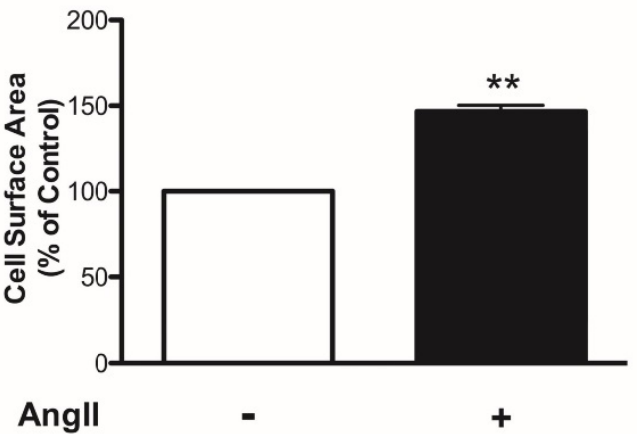

Figure 1. Cell surface area of cardiomyocytes in vitro and in vivo. (A) Representative fluorescence microscopy images of sarcomere organization (200×magnification). (B) Summarized data of cell surface area of NRVMs. (C) Representative fluorescence microscopy images of hematoxylin and eosin (H\&E) staining. (D) Summarized data of cross-sectional area of cardiomyocytes. $n=6$ experiment for each group, $* * p<0.01 v s$.control (Ctl).
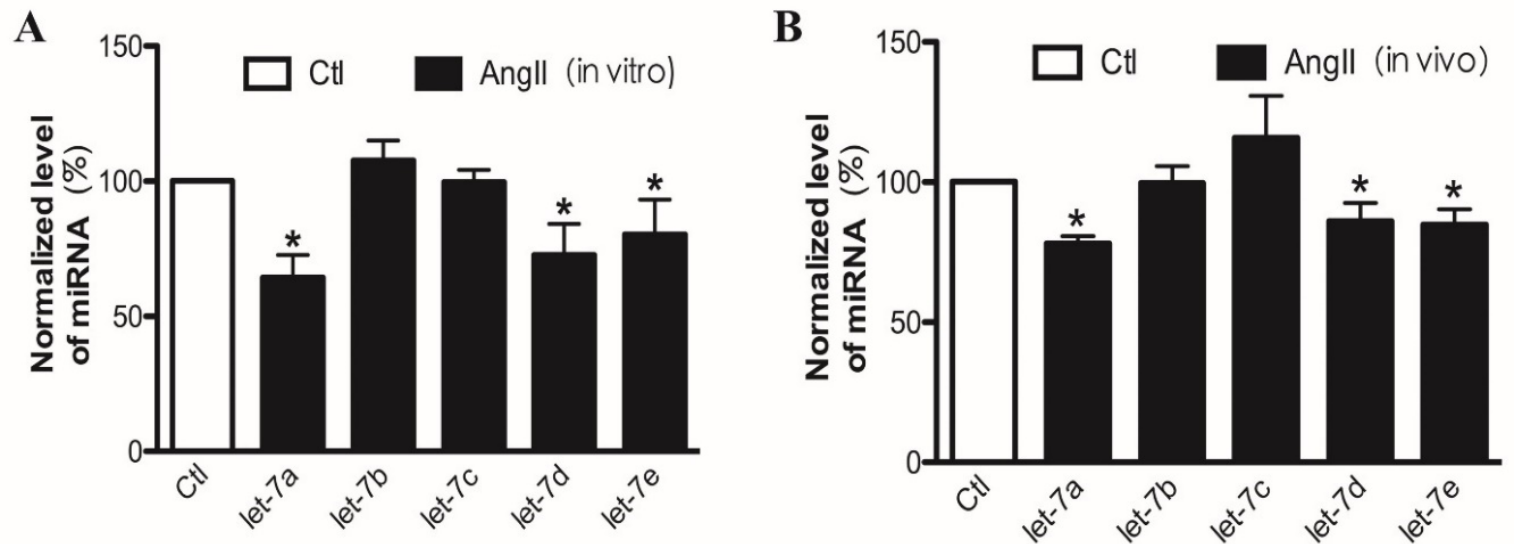

Figure 2. The expression of let-7a/b/c/d/e in cardiomyocytes treated with Angll in vitro and in vivo. Real-time $P C R$ analysis of let-7a/b/c/d/e levels in Angll-treated cardiomyocytes (A) and Angll-treated mouse hearts (B). $n=6$ experiment for each group; $* P<0.05$ vs. Ctl. 
A

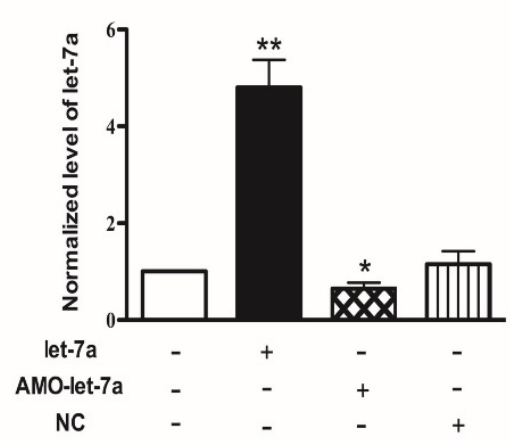

B

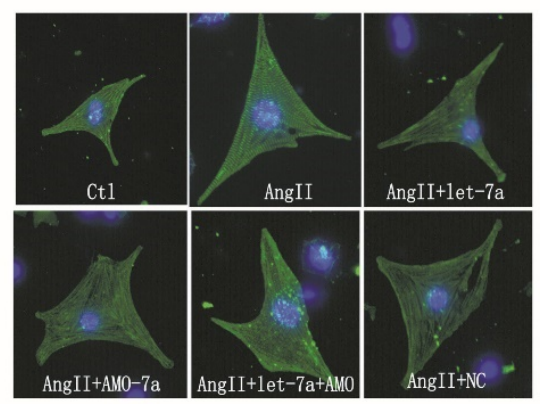

C

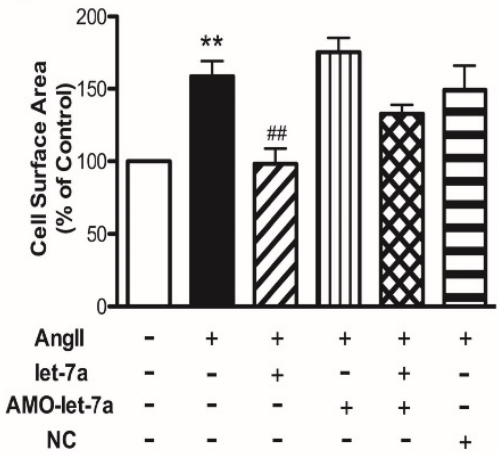

Figure 3. Effects of let-7a on cell surface area of cardiomyocytes. (A) Let-7a level in NRVMs transfected with let-7a, AMO-let-7a or NC. (B) Representative fluorescence microscopy images of sarcomere organization (200x magnification). (C) Summarized data from different treatment groups, $\mathrm{n}=60 \mathrm{cells}$ from four experiments. $\mathrm{n}=4$ experiment for each group; ${ }^{*} P<0.05 \& * * P<0.01$ vs. Ctl; $\# P<0.01$ vs. Angll.

A

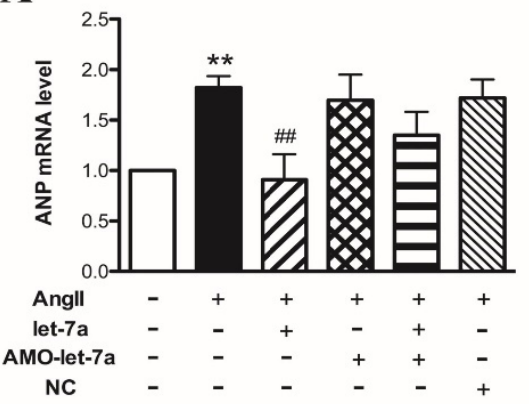

B

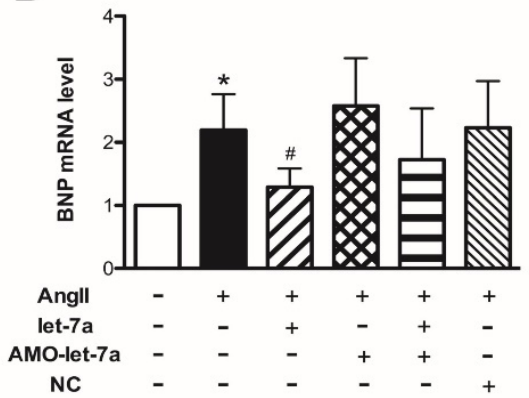

C

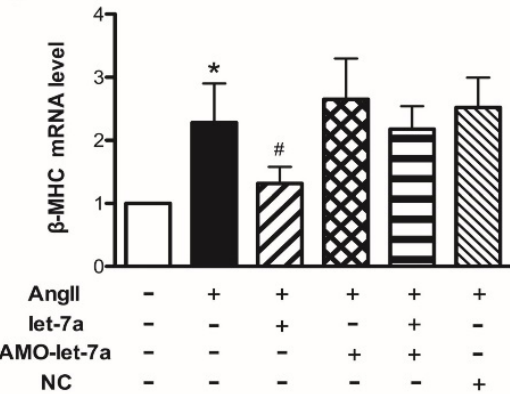

Figure 4. Effects of let-7a on expressions of hypertrophy marker genes in cardiomyocytes. The mRNA expressions of ANP (A), BNP (B), and $\beta-M H C$ (C) in different groups were detected by Real-time PCR. Data are expressed as mean \pm SD, $n=4$ experiments for each group; $* P<0.05 \& * * P<0.01$ vs. Ctl; $\# P<0.05$ \& $\# P<0.01$ vs. Angll.

\section{Overexpression of let-7a inhibited cardiomyocyte hypertrophy}

Based on the above results, we then investigated whether let-7a is involved in AngII-induced cardiomyocyte hypertrophy. We first detected the transfection efficiency of let-7a in NRVMs. Compared with control group, the level of let-7a was significantly elevated by about 5 folds in NRVMs transfected with let-7a mimics. The let-7a level was decreased by $35.5 \pm 1.3 \%$ in NRVMs treated with AMO-let-7a (anti-miRNA let-7 oligonuoleotides). The NC (negative control) had no effect on let-7a expression (Fig. 3A). We used CSA of cardiomyocytes to evaluate cardiac hypertrophy. Compared with the control group, CSA was significantly increased by $158.5 \pm 10.6 \%$ in AngII group $(P<0.01)$. Let-7a transfection could significantly decrease CSA induced by AngII; AMO-let-7a antagonized the antihypertrophic effects of let-7a; negative control (NC) of let-7a failed to do so. AMO-let-7a alone had no effect on the CSA (Fig. 3B-3C).

To further examine the effects of let-7a on cardiomyocyte hypertrophy, we tested the cardiac markers of pathological hypertrophy, atrial natriuretic peptide (ANP), brain natriuretic peptide
(BNP), and $\beta$-myosin heavy chain ( $\beta$-MHC). Our results showed that AngII significantly increased the expression of ANP (Fig. 4A), BNP (Fig. 4B), and $\beta$-MHC (Fig. 4C) at the mRNA level $(P<0.05)$. And as expected, overexpression of let-7a markedly inhibited the upregulation of these hypertrophic markers in AngII-treated cells $(P<0.05)$. AMO-let-7a abolished the effects of let-7a, and AMO-let-7a or NC alone had no effects on expression of these markers. These results indicate that let-7a produced anti-hypertrophic effects in cardiomyocytes.

\section{Camodulin 1 gene was a target of let-7a}

To elucidate the molecular mechanism of antihypertrophic effect of let-7a, we first performed Targetscan analysis to predict the possible target gene of let-7a. We found that Camodulin1 (CaM1) gene contains the "seed site" of let-7a in its 3'UTR, which is highly conserved among the species of human, rat, and mouse (Fig. 5A).

To confirm that let-7a directly targets the 3'UTR of CaM1, we constructed a chimeric luciferase reporter gene vector containing CaM1 3'UTR with the binding sites for let-7a. As shown in Fig. 5B, overexpression of let-7a resulted in significant reduction of luciferase activity. AMO-let-7a reversed 
inhibitory effect by let-7a. Neither NC nor AMO-let-7a alone affected the luciferase activity. However, co-transfection of let-7a and mutant CaM1 3'UTR did not influence the luciferase activity. These results indicated that CaM 1 gene is a direct target site of let-7a.

Western blot results showed that transfection of let-7a reduced the CaM protein level in cardiomyocyte. AMO-let-7a abolished the effects of let-7a. NC or AMO-let-7a alone had no effect on CaM expression (Fig. 6A and 6B). In addition, we demonstrated that let-7a also decreased the expression of $\mathrm{CaM}$ in cardiomyocytes treated with AngII. AMO-let-7a reversed the effect of let-7a. However, AMO-let-7a or NC did not influence CaM level (Fig.6C and 6D).

\section{Let-7a inhibited cardiac hypertrophy in vivo}

Lentivirus vectors containing pre-let-7a, A

Position 479-485 of human CALM1 3'UTR 5' ... CAUUUAAAAUUGGUUUACCUCAG .... 3' Position 474-480 of rat CALM1 3'UTR 5' ... CAUUUAAAAUUGGUUUACCUCAG ... 3' Position 472-478 of mouse CALM1 3'UTR 5 '... CAUUUAAAAUUGGUUUACCUCAG ... 3'

hsa-/rno-/mmu-let-7a hsa-/rno-/mmu-let-7b hsa-/rno-/mmu-let-7c hsa-/rno-/mmu-let-7d hsa-/rno-/mmu-let-7e hsa-/rno-/mmu-let-7 hsa-/rno-/mmu-let-7 hsa-/rno-/mmu-let-7i

$$
\|\| \|
$$

3' UUGAUAUGUUGGAUGAUGGAGU 5, 3' UUGGUGUGUUGGAUGAUGGAGU 5 ' 3' UUGGUAUGUUGGAUGAUGGAGU 5' 3' UUGAUAUGUUGGAUGAUGGAGA 5, 3', UUGAUAUGUUAGAUGAUGGAGU 5' 3' UUGACAUGUUUGAUGAUGGaGU 5, 3' UUGUCGUGUUUGAUGAUGGAGU 5 , 3' UUGAUAUGUUGGAUGAUGGAGU 5 ,
AMO-let-7a, or NC were injected into the left ventricular chamber by blocking aorta of short duration. After 2 weeks, we measured the ratio of $\mathrm{HW}$ or LVW to BW and cross-sectional area of cardiomyocytes, which are indicators of cardiac hypertrophy. As shown in Fig. 7, the ratios of $\mathrm{HW} / \mathrm{BW}$ and LVW/BW were significantly increased in AngII-treated mice, compared with the control group. Cross-sectional area of cardiomyocytes was also significantly increased in AngII-treated mice. Len-let-7a-infected mice exhibited significant inhibitory effects on the AngII-induced cardiac hypertrophy, including decrease in the $\mathrm{HW} / \mathrm{BW}$ and $\mathrm{LVW} / \mathrm{BW}$ ratios (Fig. $7 \mathrm{~A}$ and $7 \mathrm{~B}$ ) and in the cross-sectional area (Fig. 7C and 7D), which were abolished by co-infection with len-AMO-let-7a. Len-AMO-let-7a or NC alone did not affect the indicators in hypertrophic hearts.

\section{B}

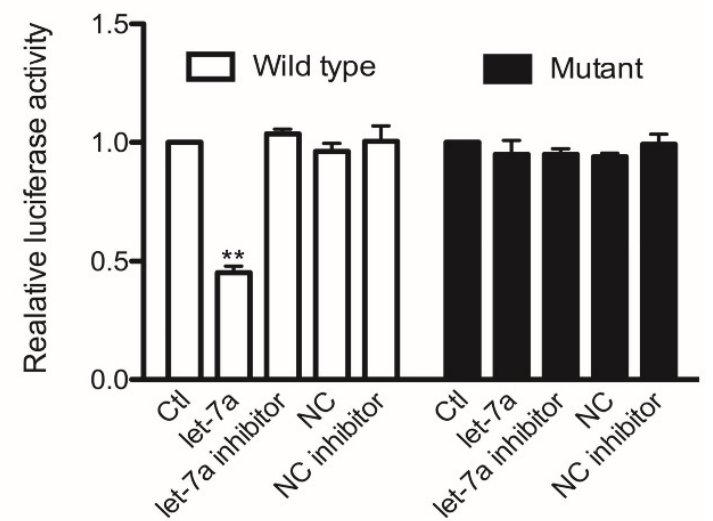

Figure 5. The regulatory effects of let-7 on Camodulin 1 gene. (A) Predicted seed-binding sites of let-7 in CaMI 3'UTR. (B) Activities of PMIR-REPORTTM luciferase vector carrying luciferase gene and fragment of CaMI 3'UTR containing the binding sites of let-7a. Data are expressed as mean $\pm S D, n=3$ experiments for each group; $* * p<0.01$ vs. Ctl.

A

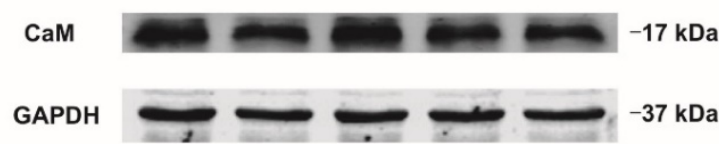

B

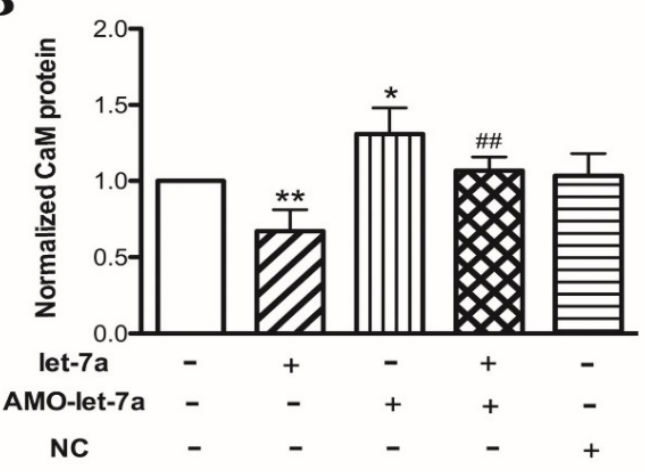

C

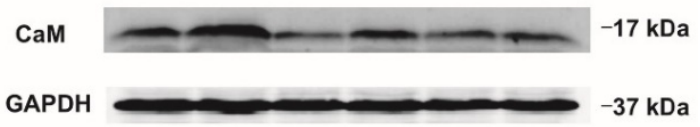

D

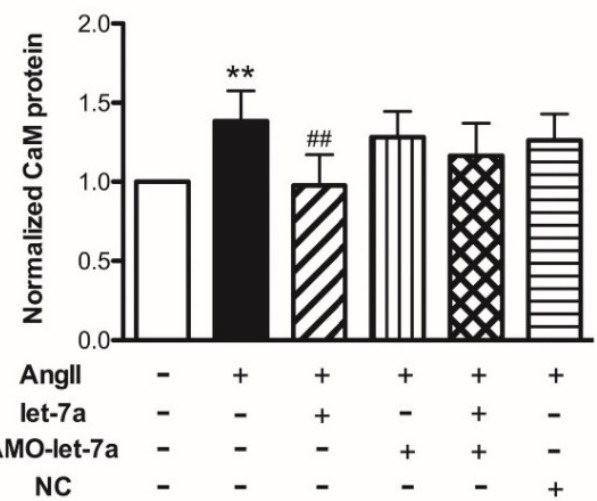

Figure 6. Effects of let-7a on calmodulin expression. Representative examples of Western blot bands from different treatments (A and C). Effects of let-7a on CaM at protein level in cardiomyocytes treated without (B) or with Angll (D). Data are expressed as mean \pm SD, $n=3$ experiments for each group; $* P<0.05 \&$ $* * P<0.01$ vs. Ctl, \#P<0.01 vs. Angll. 

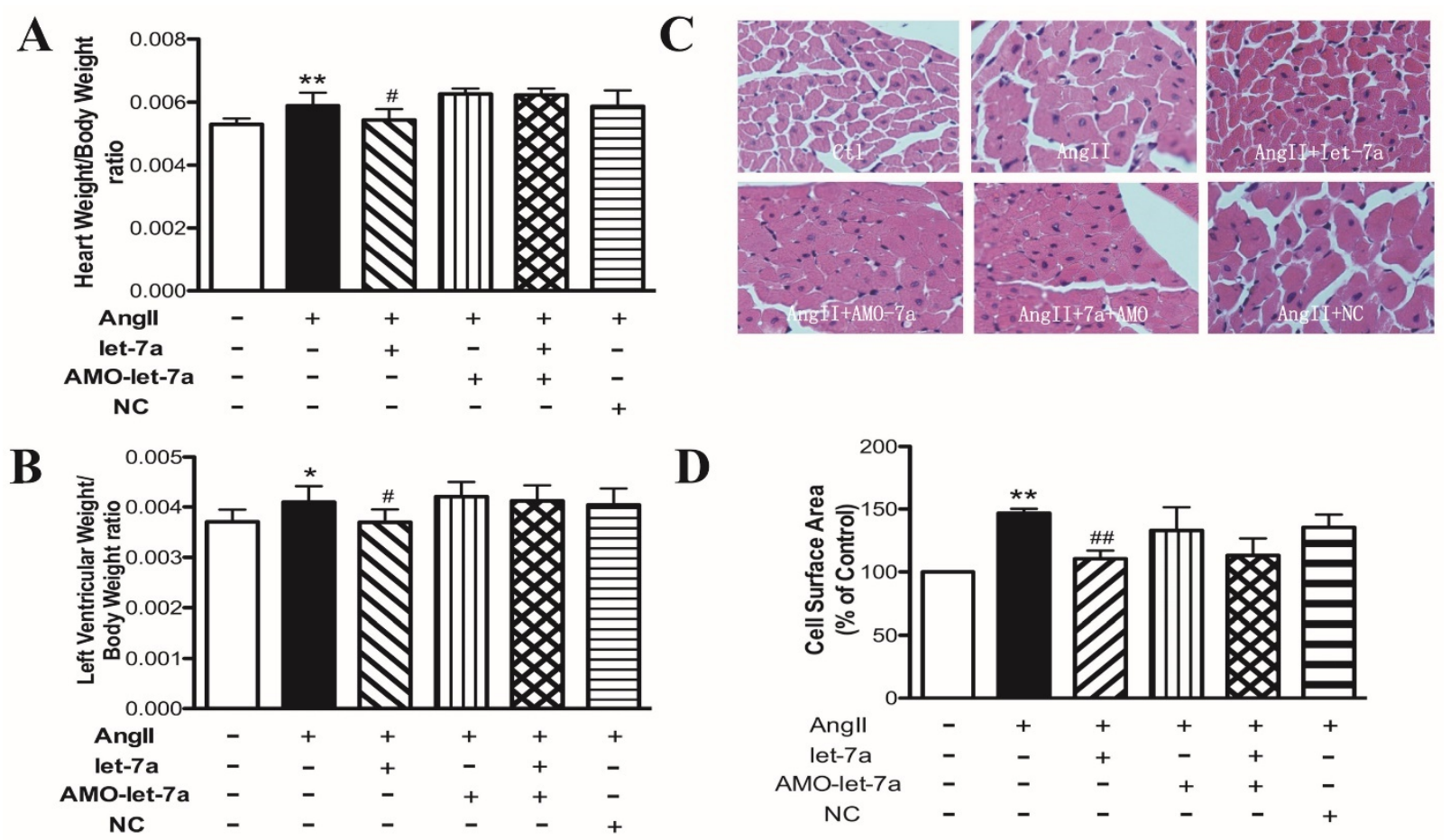

D

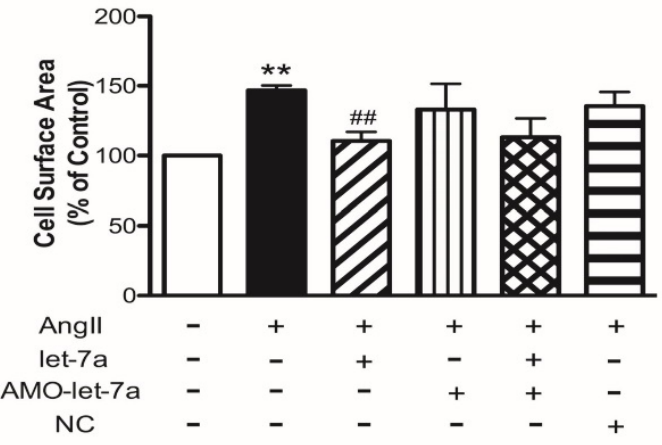

Figure 7. Anti-hypertrophic effects of let-7a in mouse model of cardiac hypertrophy induced by Angll. Ratios of HW/BW (A) and LVW/BW (B) in different groups of mice. (C) Representative fluorescence microscopic images (400× magnification) of hematoxylin and eosin (H\&E) staining. (D) Summarized data of cross-sectional area in different groups. $\mathrm{n}=6-8$ mice in each group; $* P<0.05 \& * * P<0.01$ vs. Ctl; $\# P<0.05 \& \# P<0.01$ vs. Angll.

A

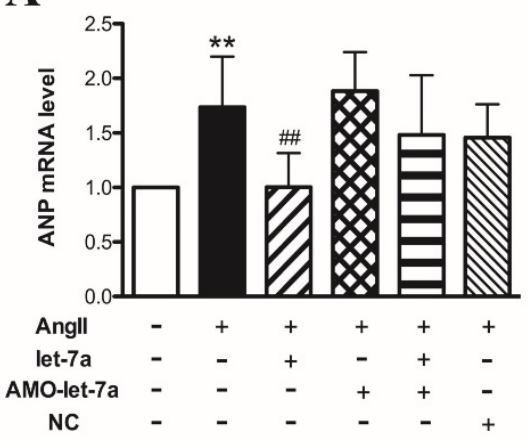

B

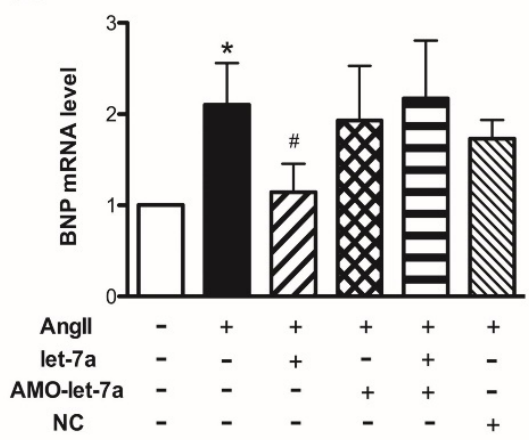

C

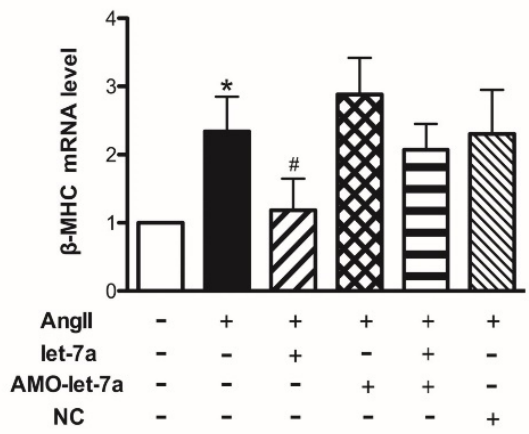

Figure 8. Effects of let-7a on hypertrophic markers in mouse model of cardiac hypertrophy induced by Angll. Expressions of ANP (A), BNP (B), and $\beta-M H C$ (C) at mRNA level in mice with different treatments. $n=6$ mice for each group, $* P<0.05 \& * * P<0.01$ vs. Ctl; $\# P<0.05 \& \# P<0.01$ vs. Angll.

Then we detected the cardiac hypertrophy markers ANP, BNP, and $\beta$-MHC mRNA levels by Real-time PCR. We found that three hypertrophy markers were upregulated in left ventricular tissues in AngII-treated mice $(P<0.05)$. Len-let-7a suppressed the AngII-induced upregulation of ANP, BNP, and $\beta$-MHC expression. However, len-AMO-let-7a prevented the effects of len-let-7a. Len-AMO-let-7a or len-NC alone had no effects in AngII-treated mice (Fig. 8).

Western blot results showed that CaM was remarkably increased at protein level in AngII group, and len-let-7a effectively inhibited this increase. Len-AMO-let-7a prevented the action of len-let-7a. Len-AMO-let-7a or len-NC alone had no effects in
AngII-treated mice (Fig. 9). These results indicate that let-7a has anti-hypertrophic effects in vivo by targeting the CaM.

Echocardiogram was performed on mice 14 days after treatment with len-pre-let-7a, len-pre-let-7a + len-AMO-let-7a, len-AMO-let-7a or len-NC. As shown in Table 2, interventricular septum depth (IVSD) and diastolic left ventricular posterior wall thickness (LVPWD) were increased in AngII-treated mice $(P<$ $0.05)$, compared with the control group. Len-let-7a significantly reversed the AngII-induced increase of IVSD and LVPWD. EF and FS values were significantly reduced in AngII-treated mice $(P<0.05)$. The EF and FS values were slightly increased in len-let-7a, but these values did not reach statistical 
significance. There were no significant changes in groups of len-pre-let-7a + len-AMO-let-7a, len-AMO-let-7a or len-NC.

In addition, we applied len-pre-let-7a, len-AMO-let-7a or len-NC to the normal mice (without AngII treatment) and found no significant changes in IVSD, LVPWD, EF, and FS. These data indicate that let-7a had no effects on the cardiac structure and function in normal condition (Table 3).

A

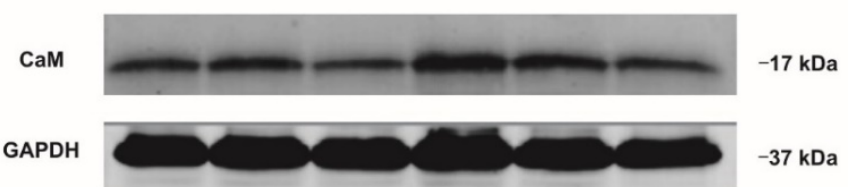

B

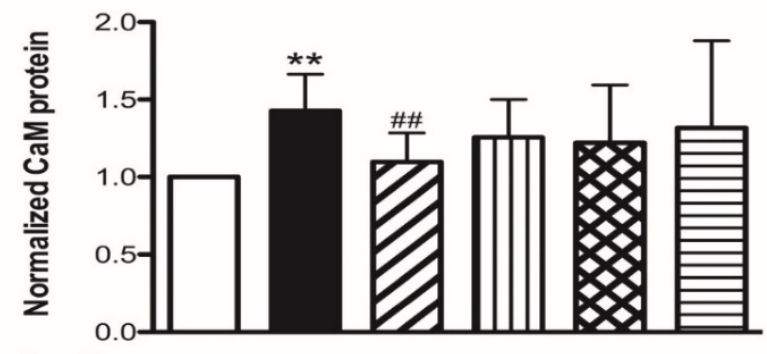

$\begin{array}{ccccccc}\text { Angll } & - & + & + & + & + & + \\ \text { let-7a } & - & - & + & - & + & - \\ \text { AMO-let-7a } & - & - & - & + & + & - \\ \text { NC } & - & - & - & - & - & +\end{array}$

Figure 9. Effects of let-7a on the expression of CaM in mouse model of cardiac hypertrophy induced by Angll. Representative examples of Western blot bands from different treatments (upper). Mean data of band density of CaM in different groups (lower). $\mathrm{n}=6 \mathrm{mice}$ in each group, $* * P<0.01$ vs. Ctl; \#P< 0.01 vs. Angll.

Table 2. Effects of let-7a on cardiac structure and function in Angll-treated mice.

\begin{tabular}{ccccccc}
\hline Group & Sham & AngII & $\begin{array}{c}\text { AngII } \\
\text { +let-7a }\end{array}$ & $\begin{array}{c}\text { AngII } \\
\text { +AMO-let-7a }\end{array}$ & $\begin{array}{c}\text { AngII } \\
\text { let-7a+AMO }\end{array}$ & $\begin{array}{c}\text { Ang II } \\
+ \text { NC }\end{array}$ \\
\hline IVSD & $1.0 \pm 0.2$ & $1.3 \pm 0.2^{*}$ & $1.0 \pm 0.1^{\#}$ & $1.1 \pm 0.2$ & $1.1 \pm 0.2$ & $1.1 \pm 0.1$ \\
LVIDD & $3.2 \pm 0.3$ & $3.1 \pm 0.3$ & $3.1 \pm 0.2$ & $3.1 \pm 0.2$ & $3.2 \pm 0.2$ & $3.1 \pm 0.4$ \\
LVPWD & $0.8 \pm 0.1$ & $1.1 \pm 0.1^{* *}$ & $0.9 \pm 0.2^{\# \#}$ & $1.0 \pm 0.1$ & $1.0 \pm 0.3$ & $1.0 \pm 0.3$ \\
EF (\%) & $78.7 \pm 3.7$ & $72.7 \pm 7.4^{*}$ & $77.2 \pm 8.8$ & $78.4 \pm 5.8$ & $79.0 \pm 10.2$ & $79.7 \pm 7.4$ \\
FS (\%) & $46.3 \pm 3.9$ & $40.9 \pm 6.7^{*}$ & $45.5 \pm 9.1$ & $46.1 \pm 5.7$ & $47.4 \pm 9.3$ & $47.7 \pm 7.3$ \\
\hline
\end{tabular}

Echocardiographic parameters in different groups of mice. IVSD, interventricular septum depth; LVIDD, left ventricular internal diastolic diameter; LVPWD, diastolic left ventricular posterior wall thickness; $\mathrm{EF}$, ejection fraction; FS, fractional shortening. $\mathrm{n}=8$ mice in each group. Data are expressed by mean $\pm \mathrm{SD}$. ${ }^{*} P<0.05 \& * * P<0.01 v$. Ctl; $\# P<0.05 \& \# P<0.01$ vs. AngII.

Table 3. Effects of let-7a on cardiac structure and function in normal mice.

\begin{tabular}{ccccc}
\hline Group & Sham & Let-7a & AMO-let-7a & NC \\
\hline IVSD & $0.8 \pm 0.1$ & $0.8 \pm 0.1$ & $0.8 \pm 0.1$ & $0.8 \pm 0.1$ \\
LVIDD & $3.5 \pm 0.3$ & $3.2 \pm 0.3$ & $3.4 \pm 0.2$ & $3.2 \pm 0.4$ \\
LVPWD & $0.8 \pm 0.1$ & $0.7 \pm 0.1$ & $0.8 \pm 0.2$ & $0.8 \pm 0.1$ \\
EF (\%) & $68.0 \pm 7.3$ & $70.5 \pm 9.7$ & $67.0 \pm 5.8$ & $66.0 \pm 4.0$ \\
FS (\%) & $37.4 \pm 6.0$ & $38.4 \pm 6.4$ & $36.7 \pm 4.5$ & $35.7 \pm 3.2$ \\
\hline
\end{tabular}

Echocardiographic parameters of mice treated with len-pre-let-7a, len-AMO-let-7a or len-NC. IVSD, interventricular septum depth; LVIDD, left ventricular internal diastolic diameter; LVPWD, diastolic left ventricular posterior wall thickness; EF, ejection fraction; FS, fractional shortening. $\mathrm{n}=8 \mathrm{mice}$ in each group. Data are expressed by mean \pm $\mathrm{SD}$. 


\section{Discussion}

Cardiac hypertrophy is a complex event, which is closely related to multiple cardiac dysfunctions. It has been reported that a number of miRNAs, such as miR-1 [24], miR-133 [25], miR-21 [26] and miR-208 [27] are involved in the process of cardiac hypertrophy. In the present study, we found that let-7a expression was downregulated in AngII induced cardiac hypertrophy. Overexpression of let-7a mimics inhibited the AngII induced hypertrophic responses and suppressed expression of hypertrophic marker genes, ANP, BNP and $\beta$-MHC. Similar results were obtained from in vivo study in a mouse model of cardiac hypertrophy. We demonstrated that let-7a exerted its antihypertrophic effects through targeting 3'UTR of CaM gene.

Let-7 was first discovered in Caenorhabditis elegans [28]. Previous studies have demonstrated that let-7 is reduced in a number of the tumors, such as lung cancer and colon cancer $[29,30]$. It participates in cellular proliferation through downregulating the RAS/c-MYC at the translational level [17]. A recent study proved that let-7 has important function in the cardiac disease. Let-7 has been demonstrated enriched in the mouse heart, and upregulated in the heart failure [31]. Li et al has demonstrated that let-7 is downregulated in the acute myocardial infarction and contributes to the anti-arrhythmic efficacy [19]. Sayed's result showed that let-7b and let-7c are upregulated, but let-7d downregulated in the TAC mice [32]. This may be due to the let-7 family members' transcript from different gene clusters, showing different function in the cardiac hypertrophy.

In present study, we found that let-7a, let-7d, let-7e were downregulated in the hypertrophic model. Our study focused on let-7a, because it demonstrated the most downregulation in hypertrophic responses both in vivo and in vitro models. Other members of the let-7 family such as let-7d and let-7e were also decreased in AngII induced hypertrophic response, indicating they may exert same function in the cardiac hypertrophy process. Notably, rest member of let-7 family let-7b, let-7c did not show significant alteration. This discrepancy of let-7 family expression may be attributed to the family members' transcript from different gene clusters playing different functions in the cardiac hypertrophy. It may be due to that members of let-7 family are located on different chromosomes (genes). Previous studies have reported that upregulation of let-7a is involved in cardiac hypertrophy [20] and miR-98/let-7 mediates the antihypertrophic effect through Trx1 [22]. These studies demonstrated the anti-hypertrophic property of let-7a and miR-98/let-7, which is consistent with our results. Taken together, let-7 family members possess antihypertrophic effect through acting on different targets, indicating let-7 family is promising target for intervening cardiac hypertrophy.

The mechanism for cardiac hypertrophy is related to changes in the organization of the sarcomeric structure that induced by altered protein and gene, such as protein C, CaM [11, 33]. CaM, in conjunction with $\mathrm{Ca}^{2+}$, activates the phosphatase protein, such as calcineurin and CAMKII, which in turn stimulate hypertrophic genes transcription [34]. We used bioinformatics approach to identify $\mathrm{CaM}$ as a predicted target of let-7a. Then, we proved that transfection with let-7a mimics obviously shut down the CaM protein expression and aborted hypertrophy effect of CaM. Luciferase report assay also confirmed the direct targeting of CaM by let-7a. Our experimental data suggest that let-7a has its anti-hypertrophy effect by targeting CaM.

The study by Ikeda et al [24] showed that miR-1 was downregulated in $\mathrm{MHCa}-\mathrm{CN}$ mouse hearts. Although expression of let-7 family miRNAs was decreased, but they did not reach statistical significance in $\mathrm{MHCa}-\mathrm{CN}$ mice. Our results showed that let-7a, let-7d, and let-7e were significantly downregulated in AngII-induced cardiac hypertrophy. These indicate that difference in miRNA expression profile is related to the various pathological conditions. They demonstrated that miR-1 negatively modulates endothelin 1-induced cardiomyocyte hypertrophy by directly targeting genes CaM and Mef2 (myocyte enhancer factor 2); the current study showed that let-7a inhibited AngII-induced cardiac hypertrophy by targeting CaM gene, indicating that a single miRNA can regulate more hypertrophic genes and one hypertrophic gene can be targeted by multiple miRNAs. Both studies showed that miR-1 or let-7a did not produce significant effects on cardiac structure and function in the normal hearts.

In summary, we show that let-7a, as negative regulator to AngII-induced hypertrophy, let-7a expression is downregulated in AngII-induced cardiomyocyte hypertrophy both in vivo and in vitro models of mouse. Overexpression of let-7a can suppress AngII induced hypertrophy, and knockdown of let-7a by its AMO prevents antihypertrophic effects of let-7a. CaM, a key regulator of cardiac hypertrophy, is a direct target of let-7a. Therefore, let-7a appears to be an interesting target for the clinical intervention of cardiac hypertrophy.

\section{Acknowledgements}

This work was supported in part by Key 
Program of National Natural Science Foundation of China (No 81530010), the Funds for Creative Research Groups of The National Natural Science Foundation of China (81121003), and the National Nature Science Foundation of China (No 81370245 and 81600192).

\section{Conflicts of interest}

None.

\section{References}

1. Bernardo BC, Weeks KL, Pretorius L, McMullen JR. Molecular distinction between physiological and pathological cardiac hypertrophy: experimental findings and therapeutic strategies. Pharmacology \& therapeutics. 2010; 128: 191-227.

2. Hill JA, Olson EN. Cardiac plasticity. The New England journal of medicine. 2008; 358: 1370-80.

3. Oka T, Komuro I. Molecular mechanisms underlying the transition of cardiac hypertrophy to heart failure. Circulation journal : official journal of the Japanese Circulation Society. 2008; 72 (Suppl A): A13-6.

4. Ahmad F, Seidman JG, Seidman CE. The genetic basis for cardiac remodeling. Annual review of genomics and human genetics. 2005; 6: 185-216.

5. Barry SP, Davidson SM, Townsend PA. Molecular regulation of cardiac hypertrophy. The international journal of biochemistry \& cell biology. 2008; 40: 2023-39.

6. Lorell BH, Carabello BA. Left ventricular hypertrophy: pathogenesis, detection, and prognosis. Circulation. 2000; 102: 470-9.

7. Frey N, McKinsey TA, Olson EN. Decoding calcium signals involved in cardiac growth and function. Nature medicine. 2000; 6: 1221-7.

8. Molkentin JD, Lu JR, Antos CL, Markham B, Richardson J, Robbins J, et al. A calcineurin-dependent transcriptional pathway for cardiac hypertrophy. Cell. 1998; 93: 215-28.

9. Wilkins BJ, Molkentin JD. Calcium-calcineurin signaling in the regulation of cardiac hypertrophy. Biochemical and biophysical research communications. 2004; 322: 1178-91.

10. Obata $K$, Nagata $K$, Iwase $M$, Odashima $M$, Nagasaka $T$, Izawa $H$, et al. Overexpression of calmodulin induces cardiac hypertrophy by a calcineurin-dependent pathway. Biochemical and biophysical research communications. 2005; 338: 1299-305.

11. Passier R, Zeng H, Frey N, Naya FJ, Nicol RL, McKinsey TA, et al. CaM kinase signaling induces cardiac hypertrophy and activates the MEF2 transcription factor in vivo. The Journal of clinical investigation. 2000; 105: 1395-406.

12. Ono K, Kuwabara Y, Han J. MicroRNAs and cardiovascular diseases. The FEBS journal. 2011; 278: 1619-33.

13. Lu Y, Zhang Y, Wang N, Pan Z, Gao X, Zhang F, et al. MicroRNA-328 contributes to adverse electrical remodeling in atrial fibrillation. Circulation. 2010; 122: 2378-87.

14. Pan Z, Sun X, Shan H, Wang N, Wang J, Ren J, et al. MicroRNA-101 inhibited postinfarct cardiac fibrosis and improved left ventricular compliance via the FBJ osteosarcoma oncogene/transforming growth factor-beta1 pathway. Circulation. 2012; 126: 840-50.

15. Yang B, Lin H, Xiao J, Lu Y, Luo X, Li B, et al. The muscle-specific microRNA miR-1 regulates cardiac arrhythmogenic potential by targeting GJA1 and KCNJ2. Nature medicine. 2007; 13: 486-91.

16. Johnson SM, Grosshans H, Shingara J, Byrom M, Jarvis R, Cheng A, et al. RAS is regulated by the let-7 microRNA family. Cell. 2005; 120: 635-47.

17. Lee YS, Dutta A. The tumor suppressor microRNA let-7 represses the HMGA2 oncogene. Genes \& development. 2007; 21: 1025-30.

18. Zou Y, Chiu H, Zinovyeva A, Ambros V, Chuang CF, Chang C. Developmental decline in neuronal regeneration by the progressive change of two intrinsic timers. Science. 2013; 340: 372-6.

19. Li X, Wang B, Cui H, Du Y, Song Y, Yang L, et al. let-7e replacement yields potent anti-arrhythmic efficacy via targeting beta 1-adrenergic receptor in rat heart. Journal of cellular and molecular medicine. 2014; 18: 1334-43.

20. Tolonen AM, Magga J, Szabo Z, Viitala P, Gao E, Moilanen AM, et al. Inhibition of Let-7 microRNA attenuates myocardial remodeling and improves cardiac function postinfarction in mice. Pharmacology research \& perspectives. 2014; 2: e00056.

21. Gray C, Li M, Patel R, Reynolds CM, Vickers MH. Let-7 miRNA profiles are associated with the reversal of left ventricular hypertrophy and hypertension in adult male offspring from mothers undernourished during pregnancy after preweaning growth hormone treatment. Endocrinology. 2014; 155: 4808-17.

22. Yang Y, Ago T, Zhai P, Abdellatif M, Sadoshima J. Thioredoxin 1 negatively regulates angiotensin II-induced cardiac hypertrophy through upregulation of miR-98/let-7. Circulation research. 2011; 108: 305-13.

23. Yu SS, Cai Y, Ye JT, Pi RB, Chen SR, Liu PQ, et al. Sirtuin 6 protects cardiomyocytes from hypertrophy in vitro via inhibition of NF-kappaB-dependent transcriptional activity. British journal of pharmacology. 2013; 168: 117-28.
24. Ikeda S, He A, Kong SW, Lu J, Bejar R, Bodyak N, et al MicroRNA-1 negatively regulates expression of the hypertrophy-associated calmodulin and Mef2a genes. Molecular and cellular biology. 2009; 29: 2193-204.

25. Dong DL, Chen C, Huo R, Wang N, Li Z, Tu YJ, et al. Reciprocal repression between microRNA-133 and calcineurin regulates cardiac hypertrophy: a novel mechanism for progressive cardiac hypertrophy. Hypertension. 2010; 55: 946-52.

26. Yan M, Chen C, Gong W, Yin Z, Zhou L, Chaugai S, et al. miR-21-3p regulates cardiac hypertrophic response by targeting histone deacetylase- 8 . Cardiovascular research. 2015; 105: 340-52.

27. van Rooij E, Sutherland LB, Qi X, Richardson JA, Hill J, Olson EN. Control of stress-dependent cardiac growth and gene expression by a microRNA. Science. 2007; 316: 575-9.

28. Reinhart BJ, Slack FJ, Basson M, Pasquinelli AE, Bettinger JC, Rougvie AE, et al. The 21-nucleotide let-7 RNA regulates developmental timing in Caenorhabditis elegans. Nature. 2000; 403: 901-6.

29. Akao Y, Nakagawa Y, Naoe T. let-7 microRNA functions as a potential growth suppressor in human colon cancer cells. Biological \& pharmaceutical bulletin. 2006; 29: 903-6.

30. Esquela-Kerscher A, Trang P, Wiggins JF, Patrawala L, Cheng A, Ford L, et al. The let-7 microRNA reduces tumor growth in mouse models of lung cancer. Cell cycle. 2008; 7: 759-64.

31. Thum T, Catalucci D, Bauersachs J. MicroRNAs: novel regulators in cardiac development and disease. Cardiovascular research. 2008; 79: 562-70.

32. Sayed D, Hong C, Chen IY, Lypowy J, Abdellatif M. MicroRNAs play an essential role in the development of cardiac hypertrophy. Circulation research. 2007; 100: 416-24.

33. Hunter JJ, Chien KR. Signaling pathways for cardiac hypertrophy and failure. The New England journal of medicine. 1999; 341: 1276-83.

34. Pan ZW, Zhang Y, Mei DH, Zhang R, Wang JH, Zhang XY, et al. Scutellarin exerts its anti-hypertrophic effects via suppressing the $\mathrm{Ca}^{2+}$-mediated calcineurin and CaMKII signaling pathways. Naunyn-Schmiedeberg's archives of pharmacology. 2010; 381: 137-45. 